\title{
Kernos
}

Revue internationale et pluridisciplinaire de religion grecque antique

$23 \mid 2010$

Varia

\section{Consécration d'un enclos funéraire à Ennodia Ilias à Larisa (Thessalie)}

\section{Bruno Helly}

\section{(2) OpenEdition \\ Journals}

Édition électronique

URL : http://journals.openedition.org/kernos/1566

DOI : 10.4000/kernos. 1566

ISSN : 2034-7871

\section{Éditeur}

Centre international d'étude de la religion grecque antique

\section{Édition imprimée}

Date de publication : 1 janvier 2010

Pagination : 53-65

ISSN : 0776-3824

\section{Référence électronique}

Bruno Helly, «Consécration d'un enclos funéraire à Ennodia llias à Larisa (Thessalie) », Kernos [En ligne], 23 | 2010, mis en ligne le 10 octobre 2013, consulté le 21 avril 2019. URL : http:// journals.openedition.org/kernos/1566; DOI : 10.4000/kernos.1566 


\section{Consécration d'un enclos funéraire à Ennodia Ilias à Larisa (Thessalie)}

Résumé : Dans deux études à paraître, José Luis Garcia Ramón et moi avons proposé de nouvelles interprétations de quelques épiclèses de la déesse thessalienne Ennodia:

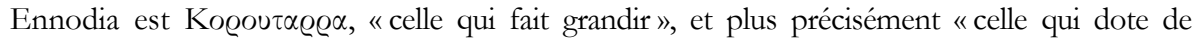
nourriture / de croissance », $\Sigma \tau \varrho o \pi \iota x \alpha$, déesse "aux éclairs », porteuse de lumière, et encore

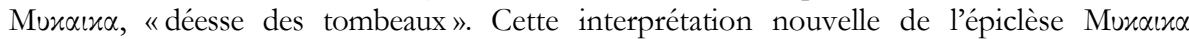
apporte un témoignage supplémentaire sur le caractère de déesse protectrice des tombeaux qu'il faut reconnaître à Ennodia. Ce caractère d'Ennodia peut être confirmé encore par la réinterprétation d'une autre inscription thessalienne, la dédicace $I G$ IX 2, 592, de Larisa. L'on peut en effet reconnaître dans ce texte fragmentaire une consécration à Ennodia d'un enclos funéraire et l'on peut y restituer de plus une autre épiclèse de la déesse, épiclèse qu'a fait connaitre une dédicace d'Azôros récemment publiée par P. Chrysostomou, pour

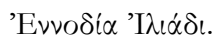

Abstract: In two studies currently awaiting publication, José Luis Garcia Ramón and I have proposed new interpretations of some epithets of the Thessalian goddess Ennodia: Ennodia is Koeoutage $\alpha$, 'the one who makes grow' and, more precisely, 'the one who provides food', $\Sigma \tau \varrho o \pi \iota x \alpha$ ' the goddess who carries lightning', and Mux $\alpha \varkappa \alpha$, 'goddess of the tombs'. This new interpretation of this last epiclesis gives us new evidence on the character of Ennodia as protectress of the tombs. This character of Ennodia can now be confirmed by the reinterpretation of another votive inscription, IG IX 2, 592, from Larisa. It is possible to recognize in this fragmentary inscription the consecration of a funerary enclosure to Ennodia; here we can make the restitution of another epiclesis of Ennodia, an epiclesis which is now seen in a votive inscription coming from Azoros in northern Thessaly and recently published, with the formula 'Evvo $\delta i \alpha$ ' $I \lambda \iota \alpha \delta$.

Dans deux études en cours de publication, nous avons engagé, José Luis Garcia Ramón et moi-même, l'examen et l'interprétation de quelques épiclèses

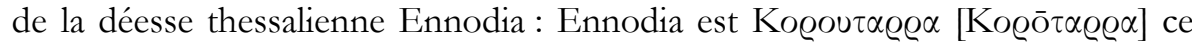
qui signifie «celle qui fait grandir», et même plus précisément "celle qui dote de nourriture / de croissance », elle est $\sum \tau \varrho o \pi \iota x \dot{\alpha}$, déesse « aux éclairs », porteuse de

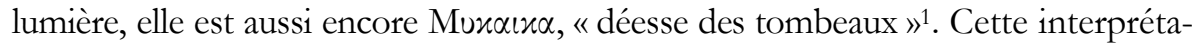

\footnotetext{
${ }^{1}$ Ces épiclèses font l'objet de deux études : 1) J.L. Garcia Ramon, B. Helly, «Evvo $\delta \propto \alpha$

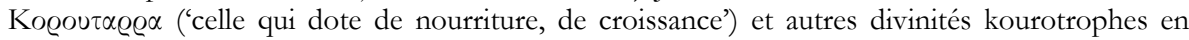
Thessalie », RPh 80, 2 (2007) [2009], p. 291-312; Eid., "Deux nouvelles épiclèses de la déesse En(n)odia dans des inscriptions de Larisa", Mélanges dédiés à Madame Francoise Bader, en

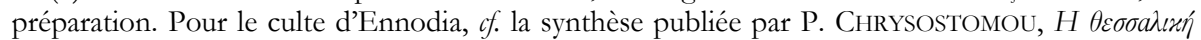
$\theta \varepsilon a ́$ Ev(v)odía $斤$ Фęaía, Athènes, 1998.
} 
tion nouvelle de l'épiclèse Mux $\alpha \varkappa \alpha$ apporte un témoignage supplémentaire sur le caractère de déesse protectrice des tombeaux $\left(\theta \eta \varkappa \alpha \iota \alpha\right.$ ou $\left.\mu u \varkappa \alpha \iota \alpha^{*}\right)$ et de divinité funéraire qu'il faut reconnaître à En(n)odia, comme le suggérait déjà une autre inscription de Larisa, la dédicace d'un temple commun à Zeus Meilichios, Ennodia et Poséidon ${ }^{2}$. Ce caractère d'En(n)odia comme protectrice des tombeaux peut être confirmé encore par la réinterprétation d'une autre inscription de Larisa, la dédicace qui a été publiée d'abord par G. Zékidis en 1901 et reprise par O. Kern dans IG IX 2 en 1908 sous le $n^{\circ} 592^{3}$. Je crois en effet que l'on peut reconnaître dans ce texte fragmentaire une consécration à Ennodia d'un enclos funéraire, inscription dans laquelle on peut restituer de plus une autre épiclèse de la déesse, épiclèse qu'a fait connaître une dédicace

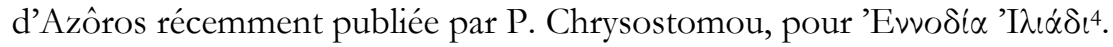

Je redonne ici le texte de la dédicace provenant de Larisa, dans la forme que lui a donnée Kern dans sa publication.

Inscription trouvée près de l'église Ag. Konstantinos (ancien quartier appelé Karagatch, sur la rue qui conduit à l'ancienne route de Volos, aujourd'hui rue du 23 octobre) à Larisa. La pierre n'a pas été retrouvée.

Bloc de calcaire bleuté, brisé à gauche, mais sans doute aussi incomplet à droite, car il semble également manquer des lettres à la fin des lignes 2-5; cependant l'incertitude subsiste sur sa forme. Dimensions prises sur l'estampage conservé à l'Académie de Berlin : $29 \times 28 \times 10$; h. 1. : 1,8 (1. 1), 1,5 - 1,8 (1. 2-8); int. : 0,8 (1. 1-2) et 0,5-0,8 (1. 2-8). Archives thessaliennes de Lyon GHW 4122.

G. Zékidis, Arch. Eph. (1901), col. 136, n 160; O. Kern, IG IX 2, 592 à partir d'un estampage qui avait été fait par Prott et dont la photographie est donnée ibidem; cet estampage est conservé au Département des Inscriptiones Graecae de la Brandenburgischer Akademie der Wissenschaften à Berlin 5; (M. Heinz, Votivstelen, A 141. Weihung / Stiftung); revu sur l'estampage (photographie Fig. 1).

${ }^{2} I G$ IX 2, 578 (cité simplement $I G$ dans ce qui suit) : je donne également le numéro de la pierre dans les archives thessaliennes de Lyon, sous la forme GHW suivi du numéro d'archivage : GHW 4108.

${ }^{3}$ IG 592 (GHW 4122).

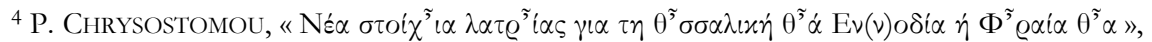

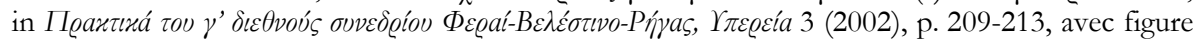
4; cf. J.-C. Decourt, B. Helly, BE 2006, 237; SEG 54 (2004), 552 (= GHW 6096). On y

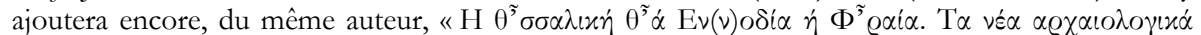

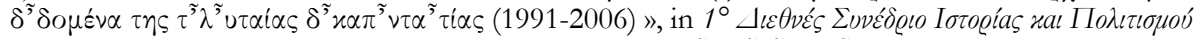

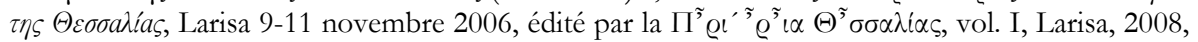
p. 244-248. Les récentes découvertes recensées dans ces publications enrichissent le volume qu'a publié P. Chrysostomou sur Ennodia, cité n. 1. Dans une étude récemment publiée, «Apollo, Ennodia and fourth-century Thessaly », Kernos 22 (2009), p. 109-124, D. Graninger tente de

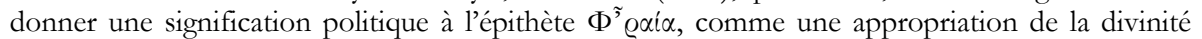
par le tyran de Phères, Jason.

${ }^{5}$ Une fois encore occasion m’est donnée de remercier très chaleureusement le Professeur K. Hallof pour son accueil et les facilités qu'il m'a offertes pour étudier les estampages d'inscriptions thessaliennes que je souhaitais pouvoir examiner lors de mon passage à Berlin en novembre 2009 . 


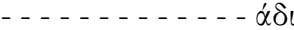

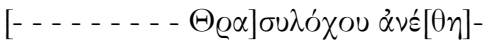

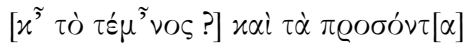

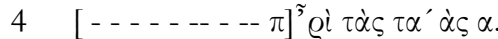

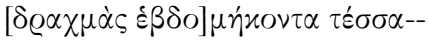

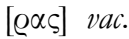

vac. $2,8 \mathrm{~cm}$

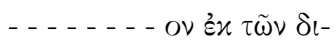

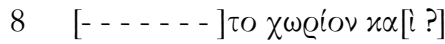

$[-.---] \operatorname{I} \nu \iota<\iota \alpha \nu$

Date : fin du II $\mathrm{e}$ s. av. (inscription non dialectale, forme des lettres) plutôt que $\mathrm{III}^{\mathrm{e}} \mathrm{s}$. av. J.-C. (Kern).

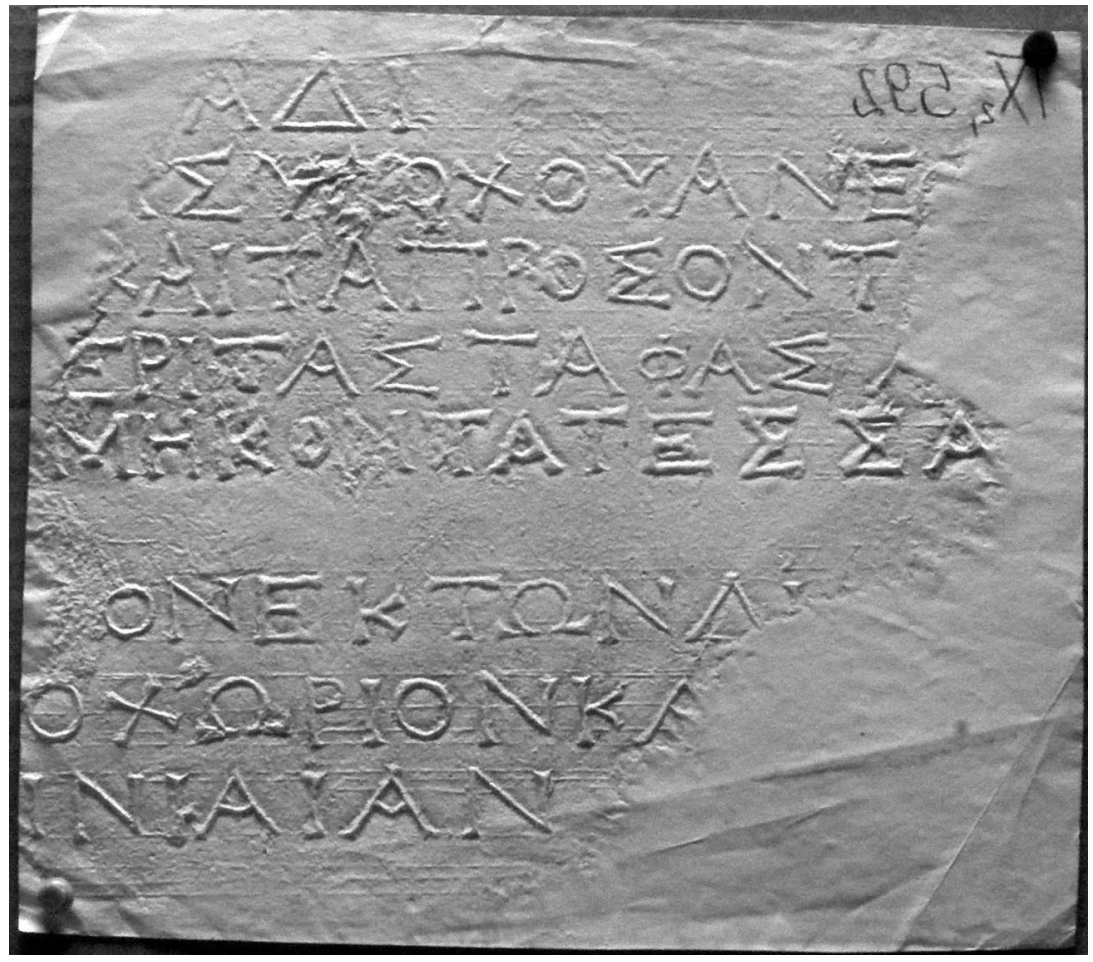

Fig. 1. Photographie de l'estampage de IG IX 2, 592 conservé à Berlin (photographie Archives thessaliennes de Lyon, avec l'aimable autorisation du Département des Inscriptiones Graecae de la Brandenburgische Akademie der Wissenschaften, Berlin).

Dans la présentation de l'inscription telle que l'a donnée O. Kern le texte des lignes 2 à 5 n'est pas en ordre : la transcription de Kern ne tient pas compte des lacunes possibles à la fin de ces lignes et ne respecte pas la coupe syllabique, qui paraît pourtant probable. Pour la compréhension même du texte, les observations 
faites par Kern ne conduisent pas à une interprétation satisfaisante non plus. Kern dit en effet dans son commentaire à la ligne 1, " exspectatur deae epitheton velut Polias, Astias », ce en quoi il a très probablement raison, au moins pour la forme de l'adjectif, mais, comme nous le verrons, les deux épiclèses qu'il propose ne conviennent pas. Kern cite à la suite une observation de Wilamowitz: "videtur dedicari domus cum horto exceptis quae deo non conveniebant sepulcris. Erasis aliis indicatur summa redituum. Infra indicatur aut id quod dedicatur aut id quod excipitur $»$. De fait les

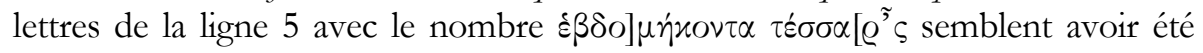
recreusées. Mais que signifie la consécration à une divinité d'un «bâtiment» (domus) et d'un jardin (bortus), et plus encore la réserve qui aurait été faite de tombeaux sur cette donation, sous le prétexte que ceux-ci ne peuvent faire partie d'une consécration à la divinité dont le nom a été perdu? On doit encore faire remarquer qu'aux lignes 4-5 le mot restitué par Kern, $\delta \varrho \alpha \chi \mu \alpha ́$, ne convient pas non plus, pour trois raisons : d'une part, à la fin de la ligne 4, le delta n'est pas assuré, mais seulement une lettre triangulaire, qui devait être suivie d'une ou deux autres lettres au moins, d'autre part la mention de drachmes n'est pas habituelle dans les inscriptions de Larisa du $\mathrm{II}^{\mathrm{e}}-\mathrm{I}^{\mathrm{er}}$ s. av. J.-C. et enfin il n'est aucunement certain que figure à cette place un prix d'achat ou de vente.

En revanche, une inscription de Nikaia dont je donne le texte ci-dessous permet d'orienter l'interprétation de $I G 592$ dans une autre direction: la consécration, non d'une maison et d'un jardin, mais celle d'un enclos funéraire. Dans l'inscription de Nikaia figurent en effet, mais sans le nom d'aucune divinité, les mêmes termes et l'expression des mêmes réalités que dans celle de Larisa : une aire réservée avec des tombes, même si les deux inscriptions ne sont pas complètement comparables, puisque le $\mathrm{n}^{\circ} 592$ est une dédicace, l'autre est une borne.

Nikaia Larisis, in situ, église Ag. Georgios (avec son cimetière) un peu hors du village.

Bloc de marbre blanc inséré dans le montant droit de la porte de l'église Ag. Georgios. Dim. : $150 \times 36,5 \times 23$; h. 1. : 1,5-2,5; int. : 1,0. Archives thessaliennes de Lyon GHW 5462.

A. Tziaphalias, AD 43 (1988) [1993], Chronika, p. 281 (SEG 43, 283); A. Kontogiannis, Nikaia Larisis. Praktika tou proton synedriou istorikon spondon tes Nikaias, 11 Aprilion 1992, Larisa, 1994, p. 44-45, nº (avec photographie pl. II, 2).

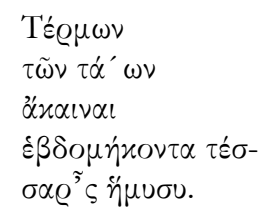

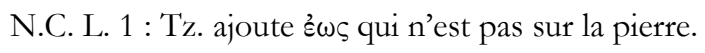

Date, selon A. Kondogiannis, ${ }^{\text {er }}$ s. av. J.-C. ou un peu plus tôt. 
Ce texte peut nous guider dans la restitution de $I G$ 592. Dans cette inscription, en effet, il s'agit très vraisemblablement d'un terrain qui a été consacré à une divinité dont le nom est perdu par un certain -N- fils de Thrasulochos ${ }^{6}$, et l'on peut bien admettre la restitution proposée par Kern de [ $\tau \dot{o} \tau \varepsilon \dot{\varepsilon} \mu^{\text {? } \nu o s] ~ a ̀ ~ l a ~}$ ligne 2, mais un autre mot, tel que í góv pourrait tout aussi bien convenir, même si ce dernier est un peu plus court. Mais l'on voit bien aussi qu'il s'agit d'un terrain dans lequel se trouvaient divers éléments, x $\alpha i$ $\tau \dot{\alpha} \pi \varrho o ́ \sigma o v \tau[\alpha]^{7}$, en relation avec des tombes, $\pi]^{\tilde{}} \varrho \grave{i} \tau \dot{\alpha} \varsigma \tau \alpha^{\prime} \alpha$ 's. Il est fréquent que l'on trouve dans les inscriptions, notamment dans les cités d'Asie Mineure, la mention d'un

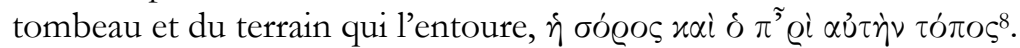

Dans plusieurs de ces inscriptions, on donne les dimensions de ces terrains, qui vont de quelques mètres carrés à plusieurs centaines ${ }^{9}$. C'est aussi une précision que l'on peut lire dans le texte de Nikaia cité ci-dessus. Ainsi, pour l'interprétation des lignes 4-5 de $I G$ 592, je trouverai, plutôt que l'indication d'un prix, celle d'une surface ${ }^{10}$, d'où, à partir de l'alpha conservé à la fin de la ligne 4, la restitution de l'unité de mesure qui commence par un alpha, $\alpha$ [ $\left[x \alpha l \mid \nu \nu \alpha_{l}{ }^{11}\right.$, suivie du nombre $\varepsilon \beta \delta o] \mu \eta \dot{\eta} \varkappa o v \tau \alpha \tau \varepsilon \dot{\varepsilon} \sigma \sigma \alpha\left[\varrho^{\tilde{F}} \varsigma\right.$, un nombre qui semble être de valeur proche sinon même identique à celle de la surface de l'enclos funéraire de Nikaia. En fait

\footnotetext{
${ }^{6}$ Le patronyme Thrasulochos, nom souvent attesté à Larisa ( $c f . L G P N, s . v$.), ne suffit pas pour identifier le dédicant.

${ }^{7}$ Pour ce participe, $c f$. la formule d'une inscription d'Égypte, par laquelle on consacre à une

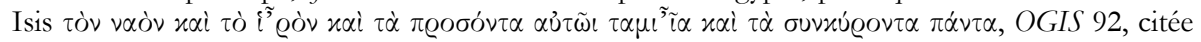
par L. RoberT, Hellenica IX, p. 47. De même dans une inscription de la vallée du Caystre, J. KeIL,

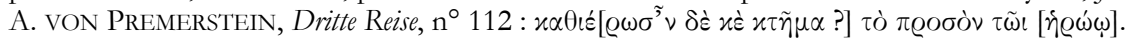

${ }^{8}$ Cf. J. KUBINSKA, Les monuments funéraires dans les inscriptions grecques d'Asie Mineure, Varsovie, 1968, p. 130, avec la série des exemples pris dans les inscriptions de Hiérapolis de Phrygie publiées par W. JudeICH, Altertümer von Hierapolis, Jahrbuch der Preuss. Akademie, Ergänzungsheft IV, 1898.
}

${ }^{9}$ Cf. Kubinska, o.c. (n. 8), p. 139-141.

${ }^{10}$ On comparera aussi le texte de la dédicace à Thémis trouvée au village moderne d'Homolio, mais qui provient du couvent Agios Dimitrios à Stomion (ancienne Rhizous), avec consécration de $\pi \varrho \circ \sigma \theta^{*} ı \tau i \delta \iota \alpha$, une avant-cour probablement, d'une surface de dix akainnai et un pied. Signalée par A.S. Arvanitopoulos, Praktika (1910), p. 192, puis par Nonna Papadimi-

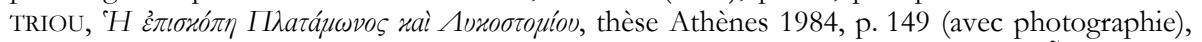

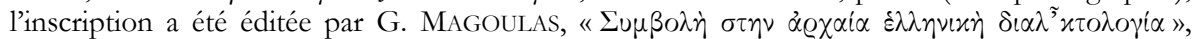
Glossologia 4 (1985), p. 89-100 avec reprise de la photographie (SEG 37 [1987], 491; cf. B. HeLLY, BE 1991, 350 (= GHW 4481, SEG 37, 491) et expliquée par J.L. GARCIA RAMON, « Neues zur Problematik des thessalischen Dialektes ", in I. Hajnal (éd.), Die altgriechischen Dialekte: Wesen und Werden. Akten des Kolloquiums Freie Universität Berlin 19.-22. September 2001, Innsbruck, 2007, p. 106109.

11 À la fin de cette 1. 4, l'estampage reproduit par Kern montre une lettre triangulaire: il devait y avoir à la suite au moins deux lettres. On ne peut cependant pas se prononcer de manière assurée sur la coupe dans le mot ¿́x $\alpha \iota v v \alpha \iota$, coupe qui devait être syllabique comme pour les autres lignes. La graphie avec deux $n u$ est bien attestée dans les inscriptions dialectales et semble nécessaire pour assurer un nombre suffisant de lettres à cette ligne (22 lettres contre 24 à la 1.3 , dont la restitution peut être considérée comme probable, même si, comme je l'ai dit ci-dessus, on pouvait avoir utilisé à cette ligne un mot plus court que $\tau \hat{\varepsilon} \mu^{5}$ vos, tel que $\hat{i}^{\tilde{}}$ Qóv). 


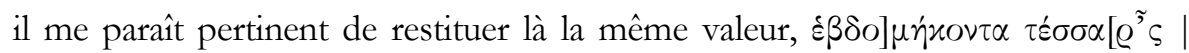
$\ddot{\eta} \mu \iota \sigma]$, en comptant l'intervalle non gravé qui aujourd'hui sépare la ligne 6 de la ligne 8 comme celui de deux lignes, dont la première serait encore occupée par un texte plus court que celui des lignes précédentes.

On peut avoir une idée de l'importance du terrain ainsi consacré dans la nécro-

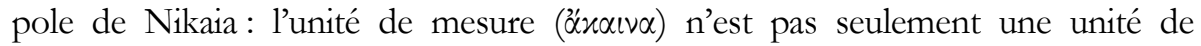
longueur valant $0,30 \mathrm{~m}$, comme l'a cru $\mathrm{A}$. Tziafalias, ce qui donnerait une longueur de $0,30 \times 74,5$ (ou 745 pieds) $=229,64 \mathrm{~m}$ (calcul mentionné dans SEG par H. Pleket, qui arrondit la valeur à $230 \mathrm{~m}$ ), mais elle est aussi une unité de surface, subdivision du plèthre ${ }^{12}$, comme l'a bien vu A. Kontogiannis : celui-ci a fait le calcul de cette surface et arrive à une valeur de $c a 708 \mathrm{~m}^{2}$, ou plus exactement un terrain d'environ $709,70 \mathrm{~m}^{2}\left(710 \mathrm{~m}^{2}\right.$ pour Pleket). Il est cependant plus intéressant d'avoir une approximation non plus de la superficie, mais des dimensions mêmes du terrain en question: partant de la mesure de surface calculée cidessus, on calcule la valeur de la racine carrée du nombre 710 , ce qui donne une évaluation de la longueur des côtés, 26,64 m $(26,64 \times 26,64=709$, 6896, soit, comme on pouvait s'y attendre, un chiffre tout proche de la valeur 709,70$)$, en supposant que le terrain ait eu la forme exacte d'un carré, ce qui n'est probablement pas le cas, mais donne un ordre de grandeur de la surface au sol à partir de la mesure du côté.

Reste à trouver ce qu'était le texte de la ligne 1, qui devait comporter un nom au datif, si l'on en juge par la finale - $\alpha \delta \iota$ seule conservée, inscrite en lettres plus grandes que le texte qui suit. Ce datif doit certainement désigner le nom du ou de

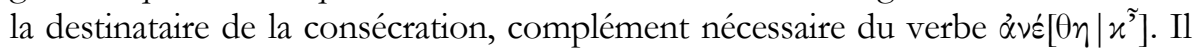
s'agit donc du nom ou de l'épiclèse d'une divinité et, de plus, d'une divinité qui pouvait se voir attribuer un enclos comportant des tombes, comme il est dit à la ligne 4, dans son périmètre : il s'agit d'un cimetière, quelle que soit sa situation, que celui-ci ne comporte que quelques tombes isolées dans la campagne ou qu'il constitue une partie d'une vaste nécropole aux portes d'une ville. Ce n'est évidemment pas n'importe quelle divinité, mais nécessairement une divinité du monde des défunts. Celle qui parait le mieux convenir ici est très probablement Ennodia, dont on sait de manière certaine qu'elle avait un sanctuaire dans la nécropole occidentale de Phères, un sanctuaire indépendant de celui au sein duquel se trouvait le grand temple qui lui était dédié13. C'est également dans une

\footnotetext{
12 Pour ces mesures à la fois de longueur et de superficie, voir B. Helly, «À Larisa. Bouleversement et remise en ordre des sanctuaires", Mnemosyne 23 (1970), p. 250-296 et F. SALVIAT, C. VATIN, «Inventaire de terrains sacrés à Larissa », in Inscriptions de Grèce centrale, Paris, 1971, p. 8 34, avec photographie p. 9 et 12. Cf. aussi V. Misailidou-Despotidou, "A Hellenistic Inscription from Scotoussa (Thessaly) and the Fortification of the City ", ABSA 88 (1983), p. 187-217, étude à laquelle A. Tziafalias a renvoyé.

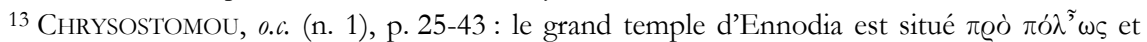
touche à la nécropole nord de la cité, le second sanctuaire, situé dans la nécropole ouest, est de moindre dimensions et n'a pas, à ce qu'il semble, le caractère monumental du précédent.
} 
nécropole de Démétrias qu'on a trouvé un sanctuaire de Pasikrata, où l'on avait fait aussi une consécration à Artémis Ennodia ${ }^{14}$.

Qu'Ennodia ait eu un rapport étroit avec les tombeaux, c'est ce que confirme aussi un passage de la Vie d'Apollonios de Tyane par Philostrate, en IV, 13: Apollonios s'embarque de Troade pour Lesbos avec une grande foule de gens et, une fois arrivé là, il renouvelle le culte que l'on consacrait localement au héros Palamède en relevant la statue de celui-ci et en consacrant autour d'elle un enclos funéraire, « de la dimension de ceux que l'on consacre pour honorer Ennodia », c'est-à-dire l'espace nécessaire pour qu'on puisse y faire un repas

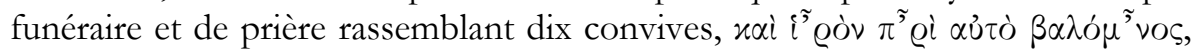

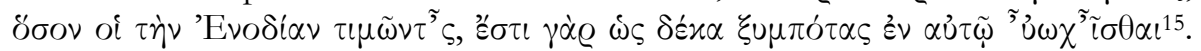
Ce texte a été bien commenté par P. Chrysostomou, dans son ouvrage de $1998{ }^{16}$, où il a conclu qu'il s'agit bien chez Philostrate de l'Ennodia thessalienne : dans la traduction, il faut donc garder le nom de celle-ci et non le remplacer, comme font certains, par celui d'Hécate. Mais nous savons aussi par Philostrate que ce sanctuaire de Palamède était établi en Éolide et qu'Apollonios en avait appris l'existence par Achille, lors de son séjour en Troade, séjour au cours duquel le

${ }^{14}$ CHrysostomou, o.c. (n. 1), p. 191-192 et pl. $26 \beta$ avec la littérature antérieure (SEG 48 [1998], 658).

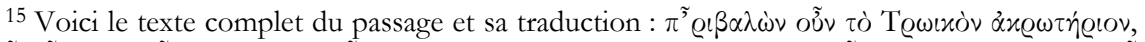

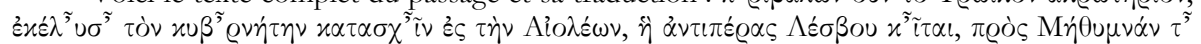

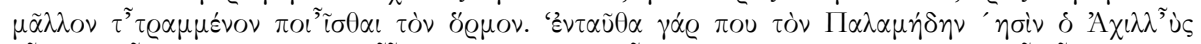

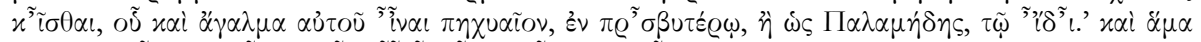

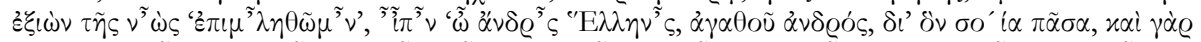

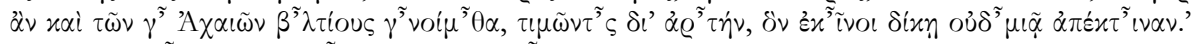

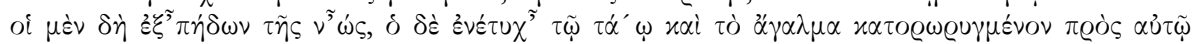

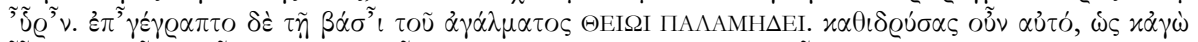

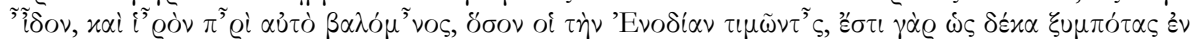

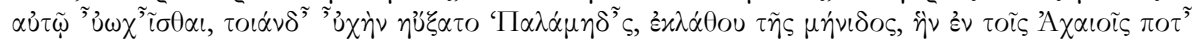

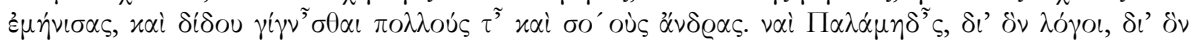

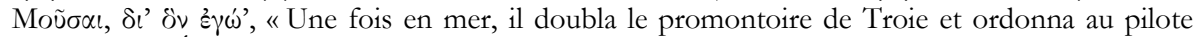
d'aborder en Éolie, en face de Lesbos, dans le port le moins éloigné de Méthymna. 'C'est là, dit-il, que repose Palamède, comme je l'ai appris d'Achille, et il y a en cet endroit une statuette du héros, haute d'une coudée, et représentant un homme au-dessus de l'âge où était arrivé Palamède.' Quand il fut arrivé, il dit en descendant à terre : 'Ô Grecs! honorons un homme de cœur de qui nous tenons toutes les sciences. Nous pourrons nous dire meilleurs que les Achéens, si nous rendons hommage au mérite d'un homme qu'ils ont mis à mort contre toute justice.' Tous les passagers mirent pied à terre : le tombeau de Palamède s'offrit aux regards d'Apollonius, et il trouva la statuette enterrée tout près de là. Il y avait sur le piédestal : AU DIVIN PALAMĖDE. Apollonius remit la statuette à son ancienne place, où je l'ai vue, consacra tout autour un espace de terre d'une étendue égale à ceux que l'on consacre en l'honneur d'Ennodia, c'est-à-dire l'espace nécessaire pour un repas rassemblant dix convives, et fit cette prière : 'Palamède, oublie la colère que tu as autrefois conçue contre les Achéens, et fais que leurs descendants soient sages en grand nombre. Exauce-moi, ô Palamède! maittre de l'éloquence, maittre des Muses, mon maitre !' » (texte et traduction, que j'ai légèrement modifiée, sur le site de l'Université catholique de Louvain : http://mercure.fltr.ucl.ac.be/Hodoi/concordances/philostrate_uita_apol_04/lecture/ 7.htm).

${ }^{16}$ Cité n. 1, p. 114-115. 
philosophe avait interrogé l'ombre d'Achille sur son tombeau ${ }^{17}$. Palamède, héros mort injustement, était un disciple du centaure Chiron, l'ami d'Achille et de Protésilaos $^{18}$ : l'anecdote se situe donc dans un contexte thessalien. Pour Philostrate, la relation avec la Thessalie, pays éolien, patrie d'Achille, était évidente ${ }^{19}$.

Nous pouvons noter d'autre part que cette histoire est un témoignage significatif sur une pratique funéraire tout à fait intéressante : celle des banquets funèbres qui se tenaient dans l'enceinte des nécropoles. Nous pouvons même tenter d'évaluer, à partir du texte de Philostrate, quelle pouvait être la surface minimale requise pour ces banquets dans les enclos funéraires : ils réunissaient en règle générale, pourrait-on dire, dix convives. Cela suppose, selon les habitudes des Anciens, de disposer d'un espace dont la superficie correspondait

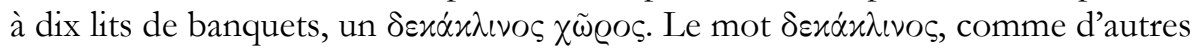
composés similaires formés sur un second élément - $\lambda \lambda \tilde{i} v o s$ précédé d'un nom de nombre, trois, neuf, dix, onze et jusqu'à trois cents, désigne une superficie équivalente à dix lits de banquet: on le trouve chez Xénophon (Écon., 8, 13),

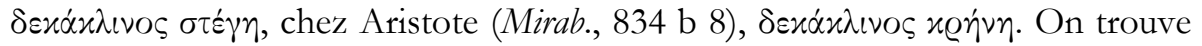
$\varepsilon \pi \tau \dot{\alpha} x \lambda \iota v o \varsigma$ dans les inscriptions : ainsi dans un acte de vente d'Amphipolis, avec

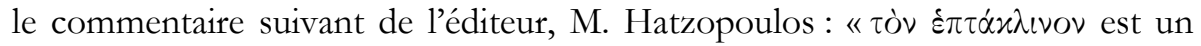

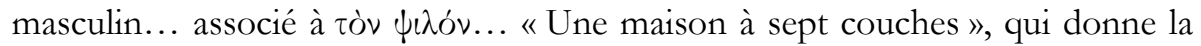
mesure de la superficie de l'andrôn, peut, par extension, servir à caractériser la maison toute entière $»^{20}$. On peut préciser le sens du mot $\varepsilon \pi \tau \dot{\alpha} x \lambda \iota v o \varsigma$ en mentionnant que deux autres attestations qui sont classiquement apportées par les dictionnaires montrent que le mot a pu servir non seulement à définir la plus ou moins grande importance d'une salle de banquet, mais aussi à indiquer par extension des surfaces de quelle que nature que ce soit ${ }^{21}$. Dans les Bélopoika de Philon de Byzance on définit des ' $\nu \lambda \alpha \varkappa \eta_{\varrho} \iota \alpha$, des protections de cuir proba-

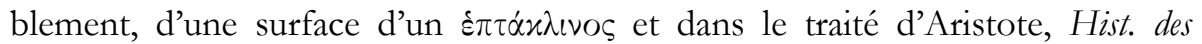

17 Cf. Philostrate, Héroikos, 53, 14-17, texte que j’ai évoqué dans mon étude d'un décret de Larisa, of. «Décret de Larisa pour le rhéteur Bombos, fils d'Alkaios, et pour Leukios, fils de Nikasias, citoyens d'Alexandrie de Troade (ca 150-140 av. J.-C.) », Chiron 36 (2006), p. 195-196.

18 Cf. Apollodore, II, 1, 5; Pausanias, X, 31, 1-2.

${ }^{19}$ Chrysostomou, o.c. (n. 1), a bien noté que Philostrate, déjà en IV, 12, le chapitre qui précède celui où il narre l'épisode du tombeau de Palamède, venait de parler, une fois encore, des Thessaliens et d'Achille.

${ }^{20}$ M. Hatzopoulos, Actes de vente d'Amphipolis, Mélétémata, Athènes, n III, 1. 4-5 (repris dans Institutions, vol. II, App. épigraphique, $\mathrm{n}^{\circ}$ 84) avec le commentaire p. 26 et les renvois à

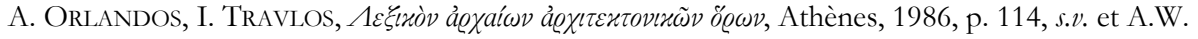
VAN Buren, s.v. «Oikos », RE XVII (1937), col. 2120-2121. On peut trouver des références archéologiques qui permettent de se faire une idée approximative des dimensions de ces pièces, $c f$. par ex. dans le palais des rois de Macédoine à Vergina: R. Ginouvès, Dictionnaire méthodique de l'architecture grecque et romaine, vol. III, Paris, 1998, s.v. « andron », p. 159, n. 97).

21 C'est le cas dans une inscription inédite de Pythion, une lettre où un certain Philoklès demande au roi Démétrios de Macédoine de lui accorder pleine possession d'un terrain décrit comme ne faisant pas plus de quatre "salles de banquets à sept lits (ou couches) », oủ $\pi \lambda \varepsilon_{\varepsilon i \omega}$ $\varepsilon \pi \tau \alpha x \lambda i \nu \omega \nu \tau \varepsilon \sigma \sigma \alpha \dot{\varrho} \omega \nu(1.7-8)$. 
animaux, dans un chapitre consacré au bison, il est dit qu'une peau de cet animal peut couvrir une surface d'un $\varepsilon \pi \tau \dot{\alpha} x \lambda \iota \nu \varsigma^{22}$. Ces textes ne nous donnent pas une mesure bien précise mais, si l'on se rapporte aux spécialistes qui commercialisent aujourd'hui des peaux de bisons d'Europe, on voit que celles-ci font couramment entre 4 et $5 \mathrm{~m}^{2}$, pour des bêtes pesant de 700 kilogrammes à une tonne, avec une hauteur au garot de $1,80 \mathrm{~m}$ au moins ${ }^{23}$. $\grave{A}$ partir de quoi on

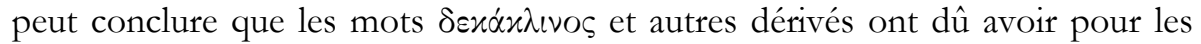
Anciens deux emplois différents, l'un pour indiquer le nombre de lits d'une salle de banquets, donc aussi indiquer, de manière relative, sa capacité d'accueil, comme nous disons aujourd'hui des salles pour quinze ou vingt convives, l'autre pour donner la mesure de la surface totale d'un espace quelconque déterminée par le nombre des banquettes pouvant accueillir un nombre bien précis de convives couchés sur ces banquettes. Dans le cas des enclos consacrés à Ennodia, il s'agissait donc de dix personnes. Si l'on prend pour base de calcul l'équivalence donnée par Aristote, on peut en déduire que la surface requise pour dix convives dans l'enceinte des enclos qui, aux dires de Philostrate, étaient consacrés à Ennodia était d'au moins 7 à $8 \mathrm{~m}^{2}$, soit approximativement un rectangle de $2,5 \times 3 \mathrm{~m}$, (pour les klinai seulement), superficie à laquelle il faut sans doute ajouter des espaces de circulation. Cela permet de donner un repère de proportion dans l'évaluation de l'enclos d'Ennodia à Larisa, environ $710 \mathrm{~m}^{2}$, mais il ne faut pas oublier que ce dernier était évidemment occupé aussi et majoritairement par des tombeaux et leur environnement.

Le texte de Philostrate apporte également une information intéressante sur une autre pratique, qui était certainement habituelle chez les Anciens, comme elle peut l'être aussi aujourd'hui, celle des visites-pélerinages, pour ainsi dire, non seulement dans les grands sanctuaires, notamment oraculaires, mais aussi dans beaucoup d'autres plus modestes : ainsi faisait Apollonios de Tyane. C'est aussi en m'appuyant sur la reconnaissance de cette pratique que je formule, dans une autre étude, une hypothèse pour tenter d'expliquer la présence d'un jeune athlète venu d'une lointaine cité d'Asie Mineure, Antioche de Pisidie, participer à des concours organisés à Larisa par la confédération thessalienne, les Éleuthéria; ce jeune garçon au nom bien attesté dans la région d'Antioche, Moas, n'était sans doute pas venu de si loin en Thessalie seulement pour courir dans ces concours, dont la réputation restait somme toute très limitée. Mais l'on sait que, dans cette lointaine cité d'Asie Mineure, se trouvait un important sanctuaire de Déméter et Koré, tout comme il en existait un en Thessalie, célèbre depuis Homère, celui de Pluton, Déméter et Koré établi sur le territoire

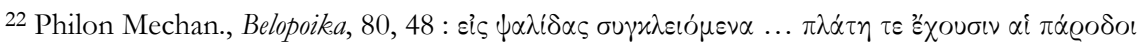

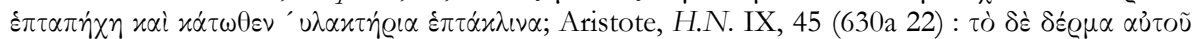

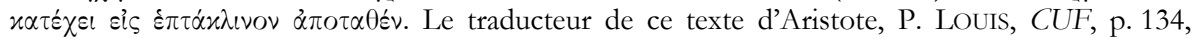

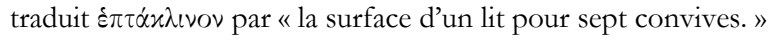

${ }^{23}$ Informations trouvées sur les sites web d'éleveurs de bisons d'Europe en Périgord, qui proposent à la vente viandes et peaux de ces animaux. 
de Leimônè, au bord de la source appelée aujourd'hui Mati, qui était considérée comme une bouche du Styx. Il me semble que telle a pu être l'objet principal du séjour en Thessalie de ce jeune Pisidien ou de ses proches ${ }^{24}$.

Dans une autre étude, encore à paraître, J.L. Garcia Ramon et moi avons tenté de mettre en évidence cette même association d'Ennodia avec les tombeaux en donnant une interprétation nouvelle d'une épithète d'Ennodia qui figure dans une inscription de Larisa faisant état d'un rapport de visite sur des sanctuaires situés hors de la ville ${ }^{25}$. Cette inscription parle d'une stèle d'Ennodia

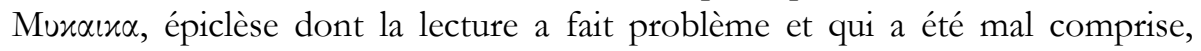
puisqu'on mettait le mot en rapport avec le verbe «mugir». Nous avons

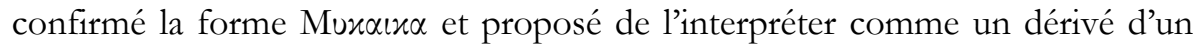
féminin $\mu u ́ x \eta$ (thess. ${ }^{*} \mu u ́ x \bar{\alpha}$ ) «boîte, cercueil » attesté dans une glose de la Souda dont l'existence est confortée par un dérivé nominal du même lexème conservé par une glose d'Hésychius : en effet $\mu u ́ x \eta$ • $\theta \dot{\eta} \varkappa \eta$ (Souda) vient en parallèle de $\mu u ́ x \omega v \cdot \sigma \omega \varrho o ́ s, \theta \eta x \omega ́ v$ (Hsch.). Il se trouve que le terme $\theta \dot{\eta} x \eta$ (thess. $\theta \varepsilon \varkappa \alpha$ ) et son dérivé thessalien $\theta \eta x \alpha i \alpha$ (avec êta notation du ē utilisée dans les inscriptions du $V^{e}-I V^{e}$ s., et qui serait dans les graphies de l'époque hellénistique $\left.{ }^{*} \theta \varepsilon \varkappa \alpha \iota \alpha\right)$ sont attestés par plusieurs inscriptions de Thessalie; nous en avons conclu que l'existence de $\theta \dot{\eta} \varkappa \alpha \iota \alpha$ à côté de $\theta \dot{\eta} \varkappa \eta / \theta \varepsilon \varkappa \alpha$ donne un terme de comparaison

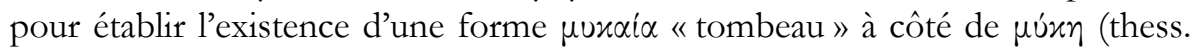
* $\mu u ̛(\bar{\alpha})$, substantif dont l'épithète Mux $\alpha \iota x \alpha$ attribuée à En(n)odia doit être un

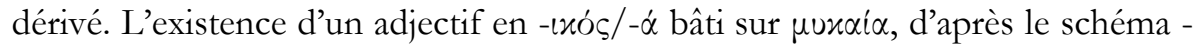

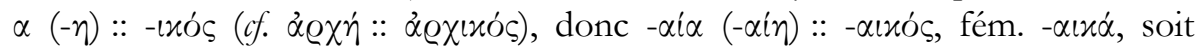
$\mu u \varkappa \alpha \iota \alpha^{*}$ [mūkaíā] 'tombeau', d'où $\mu u x \alpha \iota x o ́ s, ~ M u x \alpha \iota x \alpha$, est sans problème ${ }^{26}$. Ennodia est bien la déesse des tombeaux.

Nous avons une autre raison de restituer le nom d'Ennodia en tête de $I G$ 592, car il faut donner aussi à celle-ci une épithète qui lui convienne, en respectant la finale $-\alpha \delta$, seules lettres conservées de la première ligne, qu'il faut lui rapporter. Des adjectifs en $-\alpha \varsigma / \alpha \delta o \varsigma$, datif $-\alpha \delta$, telles que Polias ou Astias, qui ont été proposés par Kern et qui sont des épiclèses assez courantes pour d'autres divinités, ne sont cependant pas attestées pour Ennodia dans le corpus des inscriptions de Thessalie, d'après le recensement des épiclèses d'Ennodia qu'a établi P. Chrysostomou ${ }^{27}$. Mais nous avons maintenant connaissance d'une épithète 'I $\lambda_{\imath} \alpha \alpha_{\varsigma}$, attestée dans une inscription provenant d'Azôros, publiée par

\footnotetext{
24 Étude sur la liste de vainqueurs aux Éleuthéria célébrés à Larisa, IG IX 2, 529, à paraître dans ZPE (2010).

25 Cette inscription, signalée par D.R. TheOCHARIS, AD 16 (1960), Chron., p. 185 avec facsimilé pl. 158 (= GHW 3315), a fait l'objet de deux publications, l'une par Br. HELLY, l'autre par F. Salviat et C. VATIN (publications citées ci-dessus n. 12).

${ }^{26}$ Cette étude (citée supra, note 1) paraîtra dans les Mélanges offerts à Madame Françoise Bader, en préparation.

${ }^{27}$ Chrysostomou, o.c. (n. 1).
} 


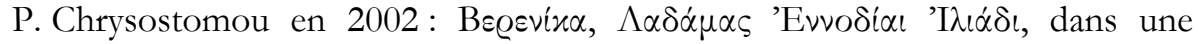
écriture que l'on peut dater du dernier tiers du IVe s. av. (= GHW 6096) ${ }^{28}$.

L'épithète 'I $\lambda \iota \dot{\alpha} \varsigma$ a naturellement retenu l'attention de P. Chrysostomou, qui a considéré que celle-ci est dérivée du nom de la ville d'Ilion et qui rappelle qu'une notice d'Étienne de Byzance a enregistré l'existence d'une Ilion en

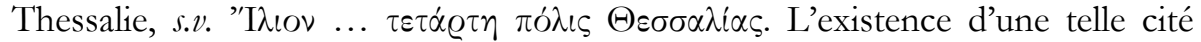
thessalienne, même dans la légende, ne me semble cependant pas établie : il ne s'en trouve aucune autre attestation et l'on sait bien qu'Étienne de Byzance a inséré dans son inventaire des noms de cités de nombreux termes qu'il pouvait prendre pour des ethniques. Mais la question de l'origine de cette épiclèse n'est pas résolue pour autant. Il existe cependant un parallèle peut-être plus éclairant; on trouve également 'I $\lambda \iota \alpha ́ s$ employé pour Athéna à Physkos en Locride orientale, dans des affranchissements par consécration ${ }^{29}$. Or il semble bien que cette Athéna Ilias doit raisonnablement être considérée comme la déesse que l'on honorait en Troade et dont le sanctuaire était le siège de la confédération des cités de cette région d'Éolide ${ }^{30}$. Il est certain en tout cas que ce culte ne pouvait pas être étranger aux cités de Thessalie, au moins à toutes celles que l'on considérait comme ayant pu faire partie de l'ancien royaume du héros Achille, puisque celui-ci avait son tombeau en Troade près du Scamandre, un tombeau sur lequel les Thessaliens, tous les Thessaliens, devaient envoyer annuellement une délégation chargée de lui rendre, selon un rituel particulier, les honneurs funèbres que le héros n'avait pas reçus dans sa patrie ${ }^{31}$. C'est donc la restitution, à la première ligne de $I G 592$, de l'épithète 'I $\lambda \_\alpha ́ \alpha$ accompagnant celle du nom d'Ennodia qui me paraît convenir le mieux à la consécration d'un enclos sacré dans lequel on trouvait aussi les tombes de quelques défunts, qui étaient peut-être des membres de la famille du dédicant, fils de Thrasylochos, probablement Larisséen.

Si telle est la bonne interprétation du document, on peut désormais présenter la première partie de l'inscription de la manière suivante :

\footnotetext{
${ }^{28}$ Chrysostomou, l.c. (n. 4).

${ }^{29} I G$ IX $1^{2}, 3,677 \mathrm{c}=$ L. DARMEZIN, Affranchissements par consécration, Nancy, 1999, p. 124-125, $\left.\mathrm{n}^{\circ} 160\right)$ et huit autres textes publiés par E. MASTROKOSTAS, SEG 16 [1959], 354-361); de nouveaux affranchissements de Physkos viennent d'être publiés par D. ROusSET, BCH 130 (2006), p. 349-379.

${ }^{30}$ Cf. L. Lerat, Les Locriens de l'Ouest, t. II, Athènes, 1952, p. 156-158 : tout en soulignant le fait que nous ne pouvons rien prouver sur ce point, l'auteur précise qu'il lui " parait impossible que les anciens n'aient pas mis ce nom en rapport avec Ilion. Athéna Ilias est le nom même de la déesse hellénistique. C'était aussi le nom de la déesse de la ville de l'Ilion hellénistique, dont la fondation était, dès le IVe siècle, attribuée à Troie » et il évoque, naturellement, l'inscription des jeunes filles locriennes, qui étaient envoyées en tribut à l'Athéna d'Ilion.

${ }^{31}$ Pour ce culte des Thessaliens sur le tombeau d'Achille, $f$. mon étude déjà citée n. 15.
} 


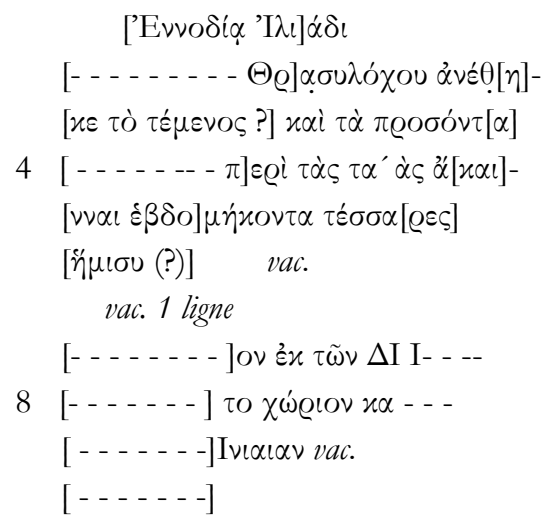

N.C. Revu sur l'estampage conservé à l'Académie de Berlin. Trois traits de réglage à toutes les lignes pour les lignes 1-5, quatre traits pour les lignes 7-9, ainsi que pour une ligne non gravée encore au-dessous. Sur les lettres, toutes les extrémités des traits sont marquées par un empattement triangulaire. La première ligne a été gravée en lettres plus grandes. L. 1 : extrémité de la haste oblique de alpha au début, de la lettre ronde (thêta) à la fin de la ligne. L. 5 : les lettres HKON sont gravées sur une rasura. L. 7 : rasura sous les lettres $n u$ et epsilon; après $\Delta \mathrm{I}$ on voit encore l'extrémité supérieure d'une haste verticale, les deux hastes pouvant bien être celles d'un êta. L. 9 : haste verticale sur le bord gauche de la feuille d'estampage, qui est rectiligne et ne couvrait donc pas toute la surface du bloc en bas.

La second partie de l'inscription est séparée de la première par au moins une ligne qui, sur la partie du bloc qui nous a été conservée, ne porte pas de texte; nous ne pouvons savoir s'il en existait un qui n'occupait pas toute la largeur de la pierre, mais seulement la partie gauche, et, si tel était le cas, nous devons considérer ce texte comme perdu pour nous. Pour les lignes qui suivent, nous n'avons aucune indication sur la longueur exacte des lignes, mais nous pouvons supposer qu'elles étaient probablement de même longueur que les précédentes. À la ligne 7, les lettres qui subsistent ne permettent pas de restituer des mots complets, mais à la fin on devait avoir un génitif pluriel qu'on pourrait peut-être interpréter comme éx $\tau \tilde{\omega} \nu \delta \eta[\mu o \sigma i \omega \nu]$. S'agissait-il d'une suite de l'inventaire de ce que renfermait l'enclos en question ou bien d'une interdiction avec une sanction ou une amende, il n'est pas possible de le déterminer. À la ligne suivante, le mot $\chi \omega$ Qíov, peut-être précédé de l'article, se lit encore en entier, mais sa signification reste indéterminée, en l'absence de contexte: faut-il le comprendre comme désignant simplement un «terrain $»^{32}$, ou lui donner un sens plus technique, celui de «forteresse, place forte», comme l'attestent de nombreuses inscriptions d'époque hellénistique: c'est un sens du terme que

32 Pour ce sens du terme, "terrain, souvent hors de la ville », cf. M.-Chr. HeLLMANN, Le vocabulaire de l'architecture dans les inscriptions de Délos, Paris, 1992, p. 207-210. 
L. Robert a plusieurs fois mis en évidence ${ }^{33}$. Si tel était le cas, nous aurions la possibilité d'interpréter aussi les quelques lettres qui sont encore conservées à la ligne 8 - INIAIAN, qu'il faut rapporter à un nom ou à un adjectif déterminatif au féminin. Comme l'inscription n'est pas dialectale, il ne parait pas possible de restituer sur ces quelques lettres une forme d'un adjectif ctétique au féminin marquant la possession, [ $\tau \dot{\alpha} \nu$ - - ]I $\nu \iota \alpha \iota \alpha \nu$, dérivé d'un anthroponyme se terminant en $-\imath \nu \iota \alpha(\varsigma)$ comme Ainias, qui pourrait donner $\left[\tau \dot{\alpha} \nu \tau \alpha^{\prime} \dot{\alpha} \nu \tau \dot{\alpha} \nu\right.$ A] $i v \iota \alpha i \alpha \nu$. Mais il existe peut-être un terme qui pourrait correspondre aux lettres qui subsistent et trouver sa place dans l'interprétation, parce qu'il peut ressortir au même champ d'organisation de l'espace que le mot de la ligne précédente, $\chi \omega$ Qiov : il s'agit du mot $\sigma \chi 0 \iota v \iota \alpha i \alpha$ qui désigne la « courtine» d'un rempart ${ }^{34}$. On pourrait supposer ainsi que l'enclos funéraire consacré par le fils anonyme de Thrasulochos à Larisa était proche du mur de quelque fortification isolée dans la campagne, voire même d'une section du rempart de la cité, dans la même situation où, à Scotoussa, se trouvaient certains enclos pour nourrir le bétail proches du

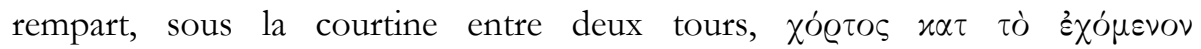
$\mu \varepsilon \sigma \alpha \pi \dot{\varrho} \gamma_{r o v}{ }^{35}$, ou encore à Larisa, un enclos au pied du bâtiment du proskénion

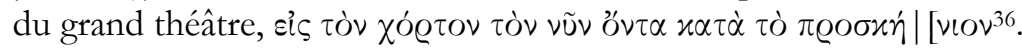

L'interprétation de l'inscription $I G 592$ présentée ici et qui vient à la suite de plusieurs autres études complète donc et renforce l'image que nous pouvons avoir de la déesse thessalienne Ennodia. Grâce aux dédicaces qui lui ont été consacrées et en dépit de la brièveté de celles-ci, le caractère d'Ennodia comme divinité du monde des morts est de plus en plus affirmé : comme Mukaika et Ilias elle protège les tombeaux, comme Koroutarra elle dote de croissance et protège les jeunes enfants, comme Stropika, qui dispense l'éclair, elle montre toute sa puissance.

Bruno HELLY

Maison de l'Orient et de la Méditerranée Jean-Pouilloux

$5-7$, rue Raulin

FR - 69007 LYON

Courriel:bruno.helly@mom.fr

\footnotetext{
${ }^{33}$ Cf. Études épigraphiques et philologiques, p. 259, n. 1 (p. 260), et surtout le compte rendu de l'ouvrage de F.G. MAIER, Griechische Mauerbauinschriften, in Gnomon (1970), p. 588-589.

${ }^{34}$ Le mot est connu par Strabon, VIII, 6, 21 et par des inscriptions d'Odessos (IPE I ${ }^{2}, 180$, 1. 4, pour une section de rempart financée par un citoyen) et d'Olbia (ibid., 32 B, 1. 38, décret pour Prôtogénès), textes repris par F.G. MAIER, Mauerbauinschriften, 1961, Ire partie, $\mathrm{n}^{\circ} 84$ et 82 , et commentaire du terme dans la II ${ }^{\mathrm{e}}$ partie, p. 81. Je remercie R. Bouchon de m'avoir signalé le texte de Philostrate (v. ci-dessus) et suggéré la possibilité de reconnaître dans l'inscription le terme spécifique attestés par ces documents.

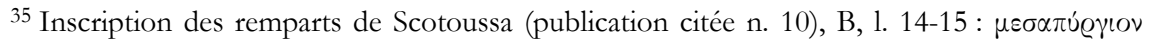
est, comme ses éléments de composition l'indiquent, la longueur de la muraille «entre deux tours ", ce que nous appelons une courtine.

${ }^{36}$ IG 522; repris par Ad. WILHELM, Gr. Inscbriften rechtlichen Inhalts, p. 37-42, pour la restitution des 1. 14-32 (SEG 13, 391).
} 